Sociologie et sociétés

\title{
Les stratégies d'intervention psychothérapeutique et psychosociale au Québec
}

\section{La régulation des conduites}

\section{Marcelo Otero}

Volume 32, numéro 1, printemps 2000

La science. Nouvel environnement, nouvelles pratiques?

URI : https://id.erudit.org/iderudit/001537ar

DOI : https://doi.org/10.7202/001537ar

Aller au sommaire du numéro

Éditeur(s)

Les Presses de l'Université de Montréal

ISSN

0038-030X (imprimé)

1492-1375 (numérique)

Découvrir la revue

Citer cet article

Otero, M. (2000). Les stratégies d'intervention psychothérapeutique et psychosociale au Québec : la régulation des conduites. Sociologie et sociétés, 32(1), 213-228. https://doi.org/10.7202/001537ar 


\section{Les stratégies d'intervention psychothérapeutique et psychosociale au Québec}

La régulation des conduites

MARCELO OTERO

Département de sociologie

Université de Montréal

C. P. 6128 , succ. Centre-ville

Montréal (Québec), Canada $\mathrm{H}_{3} \mathrm{C}_{3} / 7$

"Nous codons aujourd'hui une multiplicité de problèmes quotidiens dans le langage

psychologique, et particulièrement dans celui de la dépression, alors qu'ils étaient énoncés, il y a

encore peu, dans un langage social ou politique de la revendication, de la lutte et de l'inégalité. Cette

subjectivation généralisée est une forme sociale et politique. Elle doit être analysée comme un

processus historique et ne pas être réduite à la seule psychologisation des rapports sociaux.»

Alain Ehrenberg, (1995)

L'individu incertain, p. 23.

\section{LA NOUVELLE CONFIGURATION DU CHAMP DES INTERVENTIONS À CARACTÈRE PSYCHOLOGIQUE}

La complexité croissante du panorama psychothérapeutique actuel et l'élargissement du registre des interventions dans le domaine de plus en plus vaste de la santé mentale encouragent l'interprétation courante affirmant que ces pratiques et leurs théorisations se trouvent aujourd'hui dans un état de dispersion quasi total (Guattari, 1993, p. 5). L'émergence d'un ensemble de techniques psychothérapeutiques et de modalités d'intervention psychosociale extrêmement diversifiées ne devrait toutefois pas dissimuler le fait qu'elles présentent certains traits communs. 
L'un des plus significatifs relève du fait que les modalités contemporaines d'intervention fondent leur légitimité davantage sur leur prétendue «efficacité» que sur la justification théorique de leurs procédés ou leur inscription dans une tradition théorique prestigieuse. Cette dévaluation épistémologique de nombreuses démarches thérapeutiques au rang de techniques contribue en revanche à étendre les interventions psychologiques à ce que les gestionnaires des agences gouvernementales appellent de «nouvelles clientèles» et les praticiens privés «nouvelles demandes de services psychologiques ». ${ }^{1}$ Dans le cadre de ce processus de dévaluation-expansion, les notions de psychothérapie, intervention psychosociale, thérapie, ou service psychologique se recoupent, se permutent, voire s'équivalent, se vidant du même coup de toute signification précise, mise à part leur soi-disant contribution à la résolution de problèmes psychologiques, psychosociaux et sociaux des plus variés. En ce qui concerne les nouveaux «intervenants», ils peuvent devenir «thérapeutes» suite à une formation ad hoc jugée pertinente par des instances extrêmement diversifiées (centres de formation de toutes sortes, groupes d'auto-formation, ressources communautaires, etc.) ou s'improviser «consultants», afin de répondre à la nouvelle demande de «services psychologiques ${ }^{2}{ }^{2}$

La banalisation des discours des «experts» par les médias et la prépondérance de la culture de masse dans les sociétés occidentales ont contribué dans une large mesure à l'émergence d'un terrain fécond à la coexistence, voire au syncrétisme, des paradigmes scientifiques, pseudo-scientifiques, éthico-esthétiques, ésotériques et même religieux. En effet, les sociétés contemporaines, à l'instar des sociétés dites archaïques, semblent réactualiser la tendance à intégrer sur une même scène syncrétique les dimensions thérapeutiques et extra-thérapeutiques (corporelles, familiales, rituelles, mythiques, religieuses, esthétiques) qui ont été différenciées et cloisonnées au fil du temps par les discours analytiques des sciences (Nathan et Stengers, 1995).

Les grands récits théoriques, dont celui de la psychanalyse, de même que les institutions qui les ont véhiculés, se désagrègent au bénéfice de discours plus locaux, éclectiques et provisoires qui s'articulent à leur tour sur des mécanismes d'interpellation sociale plus flexibles et «délocalisés» (Otero, 1999). Dans ce contexte, les nouvelles techniques psychologiques se présentent comme des formules pratiques de gestion de la vie des sujets, se trouvant à mi-chemin entre la thérapeutique et le dispositif de dressage social. Au cours des années 1980, on recense déjà plus de 400 techniques dites psychothérapeutiques (Sinelnikoff 1993; Duruz, 1994), et cette prolifération se poursuit

1. En ce qui concerne le profil du «nouveau client», les études d'Alain Ehrenberg (1998) sur l'individu déprimé et de Julia Kristeva (1993) sur les «nouvelles maladies de l'âme» fournissent des pistes intéressantes pour l'analyse. Les caractéristiques essentielles des «nouvelles clientèles» cibles des interventions dans le cas du Québec sont étudiées dans notre thèse de doctorat (Otero, 2000).

2. Pour un aperçu des conflits causés par la prolifération de «consultants» et de "psy» en tout genre, les articles portant sur la polémique entourant les «actes réservés en psychologie», publiés dans la revue de l'Ordre de Psychologues du Québec, sont fort instructifs (entre autres, Maurice, 1995; Fernandez, 1997 et Bordeleau, 1998). 
dans les années 1990 (Bergin et Garfield, 1994) amplifiée notamment par le fait que les «thérapeutes» de toutes sortes font l'essai à leur gré d'amalgames particuliers en cabinet privé, en milieu «communautaire» ou en institution. L'époque des alliages scrupuleux entre l'or et le cuivre est bel et bien révolue, car l'éclectisme thérapeutique ne constitue plus une preuve de souplesse de la part des praticiens vis-à-vis de certains cas particuliers, mais il est devenu, au cours des années 1980, un véritable courant psychothérapeutique en soi (Garfield, 1984).

Si jadis les psychothérapies n'étaient susceptibles d'opérer, du moins pour l'essentiel, que sur les dimensions des sujets perçues comme "pathologiques», elles se présentent aujourd'hui comme un instrument légitime pour atteindre certains objectifs de nature extra-thérapeutique. S'émancipant en quelque sorte des contraintes diagnostiques posées par la dichotomie normal-pathologique, les interventions peuvent s'ajuster plus librement à de nouvelles situations ainsi que mettre en œuvre des stratégies d'ordre préventif, proactif ou, tout simplement, de promotion de certains comportements perçus comme plus «adaptés». Les agences gouvernementales peuvent ainsi intervenir avec une légitimité renouvelée auprès des «clientèles à risque» dans le but d'endiguer, et si possible d'anticiper, leurs «dysfonctionnements» ou leurs «difficultés» en invoquant des valeurs positives telles la satisfaction des «vrais besoins des clients", la promotion de leur «santé mentale» ou de leur «bien-être biopsychosocial».

Des expressions telles que «guérison» ou «cure» cèdent graduellement du terrain à celles d' "apprentissage » ou "développement de nouvelles habiletés et compétences», «atteinte d'objectifs», «restructuration cognitive», « responsabilisation», «autonomie», «élimination des attitudes d'échec» ou «épanouissement de tel ou tel aspect de soi». La personne souffrante, autrefois appelée patient, se métamorphose en client, usager, bénéficiaire ou, tout simplement, «personne en difficulté». La démarche thérapeutique s'assouplit à son tour pour laisser plus de place au «client» qui devient le principal responsable du succès de l'intervention en y participant activement en dehors des entrevues ou des activités thérapeutiques. L'entourage de la personne «en difficulté» est également mis à contribution dans le cadre de nombreuses stratégies d'intervention, jouant le double rôle de partenaire (aidant naturel et dépisteur de dysfonctionnements) et de nouvelle cible de l'intervention ( «ressource à outiller»).

La tendance actuelle en matière d'intervention psychothérapeutique et psychosociale privilégie les dimensions "proactives» et «alternatives» de l'intervention (encouragement de l'autonomie du "client», satisfaction de ses «vrais besoins», promotion de sa «santé mentale», etc.) au détriment des aspects considérés comme «réactifs» et «traditionnels» (déficits à combler, traitements à suivre, etc.). Toutefois, il suffit de penser aux nombreuses stratégies d'intervention concrètes auprès des «clientèles» dites lourdes ou démunies pour constater que ce que l'on nomme l'«autonomie du client»

3. Gendreau (1988) a conçu un modèle d'analyse de la signification économique du marché de la santé mentale dans le cas du Québec par le biais de l'étude des stratégies des différents «agents et promoteurs» du système concurrentiel en place. Il serait très intéressant de mettre à jour ce modèle avec les données actuelles. 
se résume souvent à une sorte d' «autonomie par défaut» (Castel 1995), voire à la «funeste liberté de celui ou celle qui n’a rien ni personne» (Laurin, 1999, p. 67).

À quoi renvoie la notion contemporaine de santé mentale dans le cadre de ces transformations? Il convient d'abord de se référer moins à l'absence de maladie qu'au développement d'une certaine capacité d'adaptation atteignable par un «travail» du sujet sur lui-même et, le cas échéant, sur son «réseau social» d'appartenance ou de «soutien». Ce travail comprend tant l'identification des besoins particuliers du sujet (les célèbres «vrais besoins du client» dans la littérature portant sur les stratégies d'intervention en matière de santé mentale) que le développement de capacités ou de ressources pour les satisfaire. Tout ce qui entrave la poursuite de ce double processus, dont la proclamée autonomie nous semble fort douteuse, est susceptible de se traduire soit par la présence de symptomatologies d'appel négatives telles l'absence de plaisir, le manque d'intérêt ou la perte de motivation, soit par des «dysfonctionnements" ou des " difficultés» majeurs, selon bien entendu la «lourdeur» de la «clientèle» dont il s'agit.

\section{L'EFFET DE RÉGULATION DES CONDUITES DES SUJETS RECONDUIT PAR LES INTERVENTIONS À VOCATION PSYCHOTHÉRAPEUTIQUE}

Nous croyons qu'au-delà des polémiques sur l'appartenance de telle ou telle technique à une certaine école plutôt qu'à une autre ou sur la prédominance de telle ou telle orientation théorique, il est plus fécond de se pencher sur la tendance à la généralisation de certains traits distinctifs caractérisant ce champ de pratiques: assouplissement théorique, délestage des dimensions historiques de la vie des sujets, action ciblée sur le " trouble» ici et maintenant, délai d'intervention minimal, ajustement personnalisé en fonction de ce qu'on nomme la demande du «client», abolition des contraintes diagnostiques nosographiques limitant l'ampleur spatiale et temporelle de l'intervention, responsabilisation et implication des «clients» et de leur «réseau» social (qu'on appelle parfois exagérément "communauté») dans la démarche thérapeutique, appel à des valeurs positives (santé mentale ou biopsychosociale, autonomie, épanouissement, etc.) pour cautionner les interventions, etc.

Vers la fin des années 1970 et au début des années 1980, certains observateurs des sociétés occidentales contemporaines ont associé l'«inflation» de la "culture psychologique» à d'autres phénomènes sociaux tels le recul des modes de socialisation axés sur la discipline (Lipovetsky, 1983), la généralisation d'un nouveau type de personnalité narcissique (Lasch, 1979) ou le repli sur la vie privée (Sennett, 1977). Sans nier le phénomène de l'émergence d'une véritable culture psychologique ou la psychologisation croissante des modes de socialisation, ces phénomènes sont loin d'être autonomes ou de l'être devenus au point de se détacher des enjeux économiques, sociaux et politiques qui structurent toute société, aussi post-moderne soit-elle. En effet, Castel (1981) affirmait à la même époque que «lorsque les options économiques, sociales et politiques, se trouvent hors des prises du sujet, le psychologique se trouve doté d'une réalité, sinon autonome, du moins autonomisée. Nous avons maintenant affaire à une subjectivité d'autant plus "libre" qu'elle ne gère plus que des enjeux dérisoires» (p. 191). 
Si l'on demande aux individus de prendre de plus en plus de responsabilités et si les frontières entre le public et le privé semblent basculer dans le sens d'un désinvestissement du premier au profit du deuxième, cela n'est pas nécessairement un symptôme d'un certain approfondissement démocratique ou d'un affranchissement des mœurs disciplinaires modernes. Plutôt qu'à une véritable «autonomisation des subjectivités», on assisterait à une nouvelle forme d'assujettissement des individus (Castoriadis, 1991), à la mise en place d'un mode de régulation des conduites qui «psychologise» en effet les rapports sociaux ou les modes de socialisation pour mieux traduire les clivages et les conflits sociaux en termes de "crise de la subjectivité», de «maladies de l'âme» ou de «manque d'habiletés et de compétences individuelles». Il n'est pas difficile de comprendre alors la place centrale qu'occupent les grilles à dominante psychologique pour interpréter et définir de nombreux "problèmes de société».

Foucault a déjà montré comment la technique de l'aveu s'est diffusée dans les sociétés occidentales, notamment à partir du xix ${ }^{\mathrm{e}}$ siècle, en investissant de nombreux aspects de la vie privée et publique des sujets, tout en fonctionnant en même temps comme une procédure régulière à l'intérieur de discours scientifiques comme ceux de la médecine, de la psychiatrie et de la psychanalyse (Foucault, 1976). Toutefois, l'injonction sociale actuelle à l'autoprise en charge de soi par la seule référence à soi suggère que les sujets sont interpellés aujourd'hui en fonction de visées différentes et que l'incitation à avouer importe moins que le développement de leur capacité d'adaptation aux contextes dans lesquels ils sont contraints d'évoluer. Rien d'étonnant alors à constater que les stratégies d'intervention psychologique traduisant le mieux cette injonction deviennent aujourd'hui les «traitements de choix».

Le complexe univers des pratiques psychothérapeutiques et psychosociales constitue un lieu privilégié tant d'observation que de gestion du social. Un lieu où se manifestent certaines injonctions sociales indiquant aux sujets ce qu'on attend d'eux; un lieu où les sujets témoignent de la résistance à ces injonctions par des symptômes, la souffrance ou le "passage à l'acte»; un lieu de reconduction de certaines «identités positives» dans lesquelles les sujets sont censés se reconnaitre; un lieu de définition de certaines conduites vis-à-vis desquelles il devient «légitime» d'intervenir. Bref, un lieu où différentes formes de régulation des conduites des sujets sont susceptibles d'être analysées.

Deux processus nous semblent fondamentaux dans tout mode de régulation des conduites, à savoir: a) la constitution des individus en «sujets» et b) l'identificationdéfinition de certaines conduites comme étant «inadaptées», "pathologiques» ou " déviantes» et de certains phénomènes comme étant des "problèmes sociaux». Les individus sont en effet quotidiennement constitués (transformés ou interpellés) en «sujets», soit en sujets assujettis à autrui (parents, supérieurs hiérarchiques, gouvernants, classes sociales, etc.), soit en sujets assujettis à leurs propres identités (sexe, profession, nation, ethnie, etc.). Si les individus n'étaient pas «constitués» en sujets susceptibles de répondre aux exigences des environnements à l'intérieur desquels ils sont contraints d'évoluer, ils ne sauraient «se conduire» de façon «adaptée». Pas de reproduction de 
clivages sociaux non plus sans constitution préalable ou simultanée des individus en sujets et, en retour, pas de sujets qui puissent fonctionner de façon «adaptée» sans l'exercice d'une forme d'intervention qui les constitue comme tels. En d'autres termes, les structures complexes de la régulation des conduites (organisations, appareils, institutions, mécanismes, pratiques, discours, etc.) assujettissent les individus en structurant le champ de leurs conduites possibles par le biais de leur constitution en sujets.

La manière dont l'être humain ou l'individu devient un sujet assujetti à la fois aux autres et à ses propres identités est intimement liée au deuxième processus que nous avons évoqué plus haut et que Foucault conçoit comme la mise en œuvre d'un type de pratiques qu'il a appelées à l'occasion "pratiques divisantes» (Foucault, 1984, p. 297). En effet, à chaque époque, dans chaque société et à l'intérieur de chaque environnement précis, les pratiques divisantes opèrent diverses formes de partage qui balisent les frontières entre le «Même» et l' «Autre», le normal et le pathologique, le conforme ou le non conforme, le phénomène social et le problème de société ou, plus près du thème de cet article, entre les conduites «adaptées» et les conduites «inadaptées».

Si l'ensemble des stratégies des interventions psychothérapeutiques et psychosociales constitue un lieu privilégié pour observer le double processus de la constitution des individus en sujets et de l'instauration d'un partage entre le "Même» et l' "Autre», en retour, c'est ce double processus de régulation qui nous permet de découvrir une certaine cohérence dans un champ de pratiques hétéroclites qu’on définit un peu trop rapidement comme chaotique.

L'individu en consultation, ou la "cible» de l'intervention, ne serait donc pas un individu au sens propre du terme mais, si l'on peut dire, un sujet de la régulation. Ce qu'on appelle aujourd'hui l'individu contemporain, l'homme incivil, l'homo psychologicus, l'individu radical ou l'individu post-moderne ne serait pas davantage " autonome» que celui issu des modes de régulation «disciplinaires» des sociétés occidentales "modernes». Si de toute évidence les formes historiques, sociales et culturelles de l'assujettissement des sujets se sont transformées, ceux-ci ne sont pas pour autant moins assujettis.

\section{L'IMPÉRATIF DE L'ADAPTATION DANS LE CONTEXTE QUÉBÉCOIS}

Il n'est plus nécessaire aujourd'hui de décortiquer les discours psychothérapeutiques de gestion des identités des sujets à l'aide de grilles d'analyse complexes qui s'acharnent à exhumer, départager, trier et dénoncer les effets de pouvoir ou les dispositifs insidieux de dressage intégrés dans le fonctionnement des discours scientifiques de vérité, car les stratégies d'intervention psychologique visent sans trop de détours l'adaptation des sujets à leur environnement.

Regardons maintenant de plus près la situation de ce champ de pratiques au Québec, notamment les orientations théoriques et les modes concrets d'intervention les plus représentatifs. ${ }^{4}$

4. La plupart des affirmations avancées dans cette partie du texte s'appuient sur l'analyse que nous avons réalisée dans le cadre de notre recherche de doctorat (Otero, 2000) à partir de l'analyse de l'ensemble 


\section{Le déclin de l'emprise de la psychanalyse et l'aggiornamento des approches d'inspiration humaniste-existentielle}

À la différence de la France et de quelques pays de l'Amérique du Sud, on peut difficilement affirmer qu'au Québec la psychanalyse a exercé dans le passé une véritable hégémonie dans le champ des pratiques psychothérapeutiques et psychosociales. Toutefois, cette «science-approche-pratique» a bien connu au Québec des époques meilleures (David 1997; Doucet et Reid, 1996).

Celle qui pour certains a été «la première thérapeutique à se donner pour objectif la liberté de l'individu, s'éloignant ainsi d'un but qui ne viserait qu'une meilleure adaptation à la société» (Richard, 1997, p. 85), se trouve aujourd'hui contrainte, elle aussi, soit à opérer son propre processus d'adaptation, soit à gérer péniblement sa décroissance. Pour les thérapeutes dont l'objectif principal n'est pas nécessairement la poursuite de l'adaptation du client, il semble difficile de concilier la satisfaction des «besoins» de celui-ci (qui sont en fait les besoins du mode de régulation sociale dominant) avec la visée plus spécifiquement analytique de la thérapie (Brillon, 1997).

Cette tension se traduit souvent par un glissement systématique des modes concrets d'intervention d'un pôle "découvrant» (centration sur l'analyse des causes profondes des symptômes) vers un pôle «recouvrant» (centration sur le contrôle des symptômes et sur l'«outillage»), et par une plus grande attention portée à certaines «clientèles» ou «populations» définies grossièrement comme «à problèmes», «à risque», «en difficulté» ou «à contraintes multiples». En effet, une observatrice de l'évolution de la psychanalyse québécoise affirme que celle-ci «est sûrement appelée à évoluer et à s’adapter, dans des conditions qui peuvent être difficiles mais aussi très stimulantes, que l'on songe à une lecture psychanalytique des processus de groupe, d'intervention auprès des femmes en difficulté, d'adolescents en crise ou de clientèles toxicomanes» (David, 1997, p. 180).

Quant aux modèles psychothérapeutiques que Castel (1979 et 1983) avait déjà baptisés «bâtards de la psychanalyse», ainsi que les innombrables bâtards de ces bâtards, l'heure est également à l'aggiornamento. Si l'on retient, dans le but de simplifier l'exposé, le label de mouvement humaniste-existentiel pour désigner cette identité thérapeutique renfermant une multitude d'écoles, de modèles et de techniques qui s'entrecoupent, s'imbriquent, et parfois, s'intègrent dans le cadre des interventions concrètes individuelles et de groupe, on peut identifier deux lignes d'évolution.

Au niveau de la pratique en bureau privé et du counseling psy médiatique (télévision, radio, Internet, journaux et revues), le courant humaniste-existentiel se développe dans toutes sortes de directions. Le panorama qui en résulte est extrêmement complexe, compte tenu de la vague d'éclectisme qui déferle depuis au moins deux décennies sur le champ des pratiques "psy» et des tentatives récurrentes d'intégration (ou de réinté-

des articles de trois publications scientifiques québécoises parues entre 1977 et 1997, à savoir: la Revue canadienne de psycho-éducation, la Revue Québécoise de Psychologie et Santé mentale au Québec: vers une nouvelle pratique. Compte tenu des contraintes d'espace imposées par la publication de cet article, nous avons limité les références bibliographiques au strict minimum. 
gration) de dimensions mystiques, ésotériques, paranormales et religieuses à certains modèles thérapeutiques, dont la revue Guide Ressources offre un échantillon éloquent (Riel et Morissette, 1984; Fernandez, 1998).

En ce qui concerne la place de ce courant-mouvement dans les milieux scientifiques, un aggiornamento s'annonce au cours de la deuxième moitié des années 1970 et s'accentue de façon significative dans les années 1980. Les approches d'inspiration humaniste-existentielle (même celles qui jouissent d'un certain prestige scientifique) perdent du terrain au profit d'autres modes d'intervention qui prétendent répondre de façon plus adéquate à ce qu'on appelle les «besoins» des clients et à ce qu'on définit comme des «problèmes de société». D'une part, le nombre d'articles scientifiques dans les revues spécialisées québécoises ${ }^{5}$ diminue et, d'autre part, on assiste à un délestage progressif des dimensions "politiques» qui accompagnaient auparavant le discours théorique de ce courant (critique de la consommation débridée, des institutions, des formes de l'aliénation des sujets, etc.) (Royer, 1977; Piotte et Duchesne, 1977; Hade-Hétu, 1978).

Au cours des années 1980, on constate dans les revues scientifiques québécoises que les activités et les démarches de croissance personnelle, l'importance du «vécu» partagé et de la relation interpersonnelle "chaleureuse», de même que la notion d'expérience, se mettent de plus en plus au service de la méta-valeur «thérapeutique» de l'adaptation des sujets à leurs environnements. En d'autres termes, on demande aux modèles d'intervention d'inspiration humaniste-existentielle de contribuer à ce qui constituait jusqu'au milieu des années 1970 l'une des causes, sinon «la» cause, de l'inhibition du "potentiel humain». Chômeurs ou «endeuillés», par exemple, peuvent trouver dans le répertoire de certaines stratégies d'intervention humaniste-existentielle des «outils» adéquats pour entreprendre le processus d'adaptation personnalisée à leur nouvelle et pénible condition (Lebeu et al. 1987; Alain et Lussier, 1988). Dans ce contexte de redéfinition identitaire, la notion de "gestion» s'intègre aisément à la phraséologie humaniste, par exemple dans la poursuite de l'objectif apparemment généreux de remettre entre les mains de la personne la gestion de son propre développement.

Rien d'étonnant à ce que l'attitude de «non directivité» et de «respect» du thérapeute envers le client laisse progressivement sa place à l'impératif pédagogique d'instrumentation du client (apprentissage de techniques spécifiques, développement d'habiletés et compétences sociales, mise en oeuvre de stratégies de résolution de problèmes) dans le but de le rendre responsable et «autonome», par exemple, dans la "gestion de ses émotions» et de ses «expériences de difficulté» (Garneau, 1984; Paquette, 1984; St-Arnaud, 1986).

La formule pour le moins amusante d'«humanisme béhavioral», qui désigne ce mariage de raison, semble trouver sa justification dans l'ineffable et populaire industrie du «faites-le vous-même», qui fait la promotion de techniques «auto-administrables» poursuivant des objectifs tels que l'auto-actualisation, l'auto-changement, l'auto-contrôle, l'auto-développement, l'auto-formation, l'auto-guérisson, etc. (Boisvert et Beaudry, 1984).

5. Par exemple, dans la Revue canadienne de psycho-éducation, la Revue Québécoise de Psychologie et Santé mentale au Québec: vers une nouvelle pratique. 


\section{Les interventions d'inspiration cognitivo-comportementale}

Les interventions d'inspiration cognitivo-comportementale ont connu une diffusion extraordinaire au cours des deux dernières décennies (Kuehlwein et Rosen, 1993; Cousineau, 1995). Parmi les principales raisons de leur succès on retient souvent leur caractère instrumental, leur simplicité théorique et leur énorme capacité d'adaptation à de nombreux contextes et situations. À l'opposé des approches d'inspiration humaniste-existentielle et d'inspiration «communautaire», qu'on évoquera plus loin, le cognitivisme (ou les approches cognitivo-comportementales en général) ne prétend pas s'insérer dans un projet ou mouvement social plus global, pour modeste qu'il soit. En ce sens qu'il n'existe pas de «mouvement» cognitif ou cognitivo-comportemental à l'instar des mouvements communautaire ou socio-communautaire, ou encore, à l'instar du mouvement humaniste (De Carvalho, 1990).

Ainsi dissociées de leur inscription dans un «mouvement» précis, les modèles d'intervention d'inspiration cognitivo-comportementale se trouvent plus à l'aise à l'heure de mettre au point ou d'intégrer des «outils» appartenant à d'autres approches ou de partager leur panoplie de techniques dans le cadre de stratégies d'intervention diverses. Qu'on pense à l' " outillage » du client et de son «réseau social» (aidants naturels, ex-clients, etc.) dans le cadre des stratégies d'intervention d'inspiration «communautaire», ou encore, à l'«instrumentation» du client dans le cadre des interventions humanistes-existentielles (St-Arnaud, 1984; Garneau, 1984).

La voie royale pour l'amélioration des habiletés et des compétences d'innombrables «clientèles» soi-disant à la recherche d'une meilleure adaptation est aujourd'hui cognitivo-comportementale. On pourrait même avancer l'hypothèse que l'éclectisme psychothérapeutique contemporain devient de plus en plus cognitivo-comportemental, compte tenu des préoccupations «instrumentales» au cœur des très nombreuses démarches thérapeutiques qui s'inspirent de sources théoriques et de traditions extrêmement variées, voire contradictoires. Si la conception et les usages plus ou moins classiques de l'intervention comportementale n'ont certes pas disparu, celle-ci s'est assouplie en même temps qu'elle a revampé son image de par son association ou arrimage aux techniques cognitives qui ne soulèvent pas les controverses éthiques du béhaviorisme classique.

Les cognitivistes considèrent que des schémas de pensée qui comportent des biais cognitifs «appris» dans les différents contextes de socialisation de la vie des sujets (famille, école, travail, médias, etc.) sont à l'origine de nombreux «symptômes» et comportements «dysfonctionnels». Situés dans la «mémoire à long terme», ces schémas cognitifs se comportent comme un «filtre» de l'information qui organise la perception sélective et, au bout du compte, l'expérience vécue des individus de façon à ce que la réalité s'y adapte en quelque sorte, et reconfirme ces schémas «dysfonctionnels» qui sont continuellement renforcés et «sur-appris».

Le thérapeute cognitif s'applique à modifier les pensées dysfonctionnelles (anticipations, idées noires, attentes irréalistes ou résignation acquise, croyances négatives et irrationnelles, inférences «incorrectes», etc.) par le biais d’une démarche pédagogique 
censée conduire le client à la prise de conscience des distorsions cognitives dysfonctionnelles et à l'apprentissage progressif d'attitudes, habiletés et compétences plus «adaptées». Dans ce processus, l'implication du client devient cruciale, car c'est lui qui doit assumer la responsabilité du changement (intégration d'un répertoire cognitif «adapté») et de la gestion des «expériences de difficulté» (ici et maintenant) qui l'ont amené en consultation. Il s'agit souvent d'inculquer aux sujets une vision stratégique d'euxmêmes et de leur environnement, qui leur permette de s'engager «efficacement» dans la résolution des problèmes concrets, d'identifier clairement «leurs» besoins, de dresser des plans d'action «réalistes » et, enfin, d'évaluer les diverses réponses du milieu afin d'effectuer la mise au point de leurs répertoires cognitifs et comportementaux. La souplesse et le pragmatisme des répertoires cognitifs et comportementaux du sujet (ou tout simplement la capacité d'adaptation) deviennent bien entendu des atouts dans un environnement instable (familial, de travail, financier, etc.) comme celui qui caractérise à plusieurs égards les sociétés actuelles.

La présence chez le client de schémas cognitifs dysfonctionnels, le manque de motivation, l'établissement de stratégies inadaptées, l'absence de "perspective future» ou la formulation de buts «irréalistes», pour ne nommer que ceux-ci, sont couramment associés à des symptômes variés (anxiété, dépression, somatisations, stress, etc.). En revanche, le choix de stratégies et de buts personnels "adéquats» ou «adaptés», et le fait d'avoir une perception «réaliste» de l'environnement et de soi-même, par exemple, constituent des facteurs de «santé mentale» (Bouffard, 1993; Bouffard et Bastin, 1993). Adaptation et santé mentale deviennent des termes interchangeables, ou plutôt, le premier est souvent présenté comme la condition de possibilité du deuxième, et ce, non seulement dans le jargon cognitiviste, mais également, comme on le verra ci-dessous, dans le jargon «communautaire».

Dans l'univers des théories psychosociales cognitives, l'allusion aux facteurs économiques, sociaux ou politiques qui pourraient avoir une part de responsabilité en ce qui concerne les «difficultés» des personnes «en difficulté» ne semble ni nécessaire ni pertinente. Le sujet auto-motivé est conçu comme le lieu d'ancrage d'un répertoire cognitivo-comportemental qui lui permettrait, s'il est convenablement adapté, d'évoluer sans heurts significatifs dans son environnement en passant successivement d'un équilibre à un autre (Bandura, 1993).

\section{Les modes d'intervention d'inspiration communautaire}

On ne fera état ici ni des origines historiques du mouvement communautaire ou sociocommunautaire, ni de la complexité et de la variété de ses nombreuses composantes (psychiatrie communautaire, psychologie communautaire, groupes sociaux, groupes d'entraide, structures intermédiaires, ressources alternatives, etc.). On se bornera à faire ressortir certaines de ses caractéristiques fondamentales dans le but de mieux comprendre la popularité actuelle des interventions communautaires.

Ce mode d'intervention n'a pas seulement connu une expansion extraordinaire au cours des vingt-cinq dernières années, mais il se présente également aujourd'hui 
comme un outil des plus flexibles, efficaces et économiques pour faire face aux tableaux socio-cliniques les plus variés. Qu'il s'agisse de la clientèle psychiatrique la plus «lourde» (vis-à-vis de laquelle les modèles cognitivo-comportemental et humaniste-existentiel semblent impuissants), de la prévention de toutes sortes de «dérapages» comportementaux, du contrôle du stress environnemental, ou tout simplement de la promotion de la «santé mentale» auprès de la population en général, les modèles d'inspiration communautaire proposent des formules d'intervention peu dispendieuses, se caractérisant par une moindre sollicitation des professionnels et du réseau de services publics.

L'appel à l'auto-prise en charge communautaire, à l'empowerment et à la responsabilisation des citoyens-clients définit une zone de dialogue et de collaboration possible avec les modèles d'inspiration humaniste-existentielle dans leur version «adaptée» des années 1980, et avec les modèles cognitivo-comportementaux, tous deux fournisseurs d'outils dits auto-administrables.

Les partisans de l'orientation communautaire d'intervention en santé mentale affirment partager une conception biopsychosociale de l'être humain évoluant dans son milieu, sa communauté ou son écosystème. De ce fait, l'objectif principal du mouvement socio-communautaire en santé mentale se définit comme étant le développement des conditions favorisant l'épanouissement de la personne dans son environnement (Tremblay, 1996). Cependant, il ne s'agit pas tant ici du développement $\mathrm{du}$ «potentiel humain» de la personne que du «potentiel d'adaptation» des sujets, qu'on prétend également inné à chaque être humain en même temps qu’indissociable de l'environnement. Compte tenu du fait que toute communauté est conçue comme une sorte de réseau complexe de besoins et de ressources, l'intervenant communautaire s'efforce de rendre compatibles les besoins particuliers des sujets avec les «ressources» dont dispose leur «communauté» respective. En d'autres termes, il tente en principe de favoriser l'adaptation mutuelle «individu-environnement».

$S$ 'il est vrai que les intervenants communautaires identifient à l'occasion ledit stress environnemental comme étant l'une des sources des «difficultés» des clients, les stratégies d'intervention concrètes définissent leurs cibles le plus souvent au niveau du développement des capacités d'adaptation (physiques, cognitives ou affectives) des sujets et de leur entourage (famille, voisins, amis, etc.) aux exigences de l'environnement. En effet, la référence à des facteurs économiques, sociaux, politiques et culturels auxquels les intervenants communautaires accordent une grande importance sur le plan rhétorique, se dilue systématiquement dans le cadre de la notion fourre-tout de stress environnemental et finit par disparaittre en cours d'intervention. Les intervenants communautaires doivent en effet être capables de repérer le "potentiel et les capacités adaptatives des clientèles» malgré les limites éventuelles de leur situation, et de mettre à profit les ressources communautaires susceptibles de répondre à «leurs» besoins, qui doivent s'adapter à leur tour, de façon raisonnable, responsable et « réaliste», aux limites de ces mêmes ressources. De ce fait, la perspective d'intervention «biopsychosociale», «écologique», "communautaire», «socio-communautaire» ou "écosystémique», susceptible de permettre une meilleure vue d'ensemble des dits problèmes de santé men- 
tale, se résume en bout de ligne à la poursuite de l'adaptation unilatérale des sujets à leur environnement lorsqu'il s'agit de mettre au point des stratégies concrètes d'intervention (Bouchard 1986).

Idéalement, les interventions communautaires sont censées opérer dans une optique proactive ou préventive primaire (Caplan 1964), au sens où elles n'ont pas nécessairement été sollicitées pour régler un problème qu'elles doivent toutefois anticiper. De façon générale, la prévention primaire se définit en termes de développement chez les sujets de capacités, compétences ou habiletés adéquates pour «faire face» ou s'adapter à leur environnement (entre autres: Perreault et Béguin, 1994). L'intervention virtuelle sur la santé mentale est considérée depuis longtemps déjà comme «l'aspect le plus progressif et le plus prometteur du mouvement de santé mentale communautaire québécois» (Campagne, 1981, p. 22), ou encore comme "une révolution en santé mentale puisqu'elle remet en question le caractère médicalisé des interventions psychothérapeutiques individuelles, mettant l'accent sur la relation dynamique personne-environnement» (Nadeau, 1983, p. 14). Si la prévention primaire vise à réduire le nombre de personnes susceptibles d'être atteintes de "problèmes de santé mentale » dans la «communauté» en général (et du même coup le nombre de clients potentiels du système de santé), la stratégie d'intervention se limite très souvent au seul renforcement de la capacité d'adaptation des sujets. Quant au caractère "progressiste», voire «révolutionnaire», de l'adaptation unilatérale des sujets (les «clients» et leur entourage) à leur environnement et à leurs «expériences de difficulté», rien ne semble plus incertain.

Mais qu'est-ce que signifie être en «santé mentale»? Cette notion fort populaire et en même temps la pièce maîtresse du dispositif d'intervention d'inspiration communautaire n'est que rarement définie de façon précise. En dépit de ce constat, de très nombreux programmes et stratégies d'intervention sont mis sur pied en son nom ou au nom de notions plus ou moins équivalentes telles que «santé mentale communautaire», «santé psychosociale» ou «qualité de vie», qui vont d'ailleurs de pair avec ladite conception biopsychosociale de la personne. À l'instar des modèles d'intervention cognitivo-comportementale, on constate que dans le cadre des modèles d'inspiration communautaire, la capacité d'adaptation constitue également la condition de possibilité de la santé mentale des sujets. C'est pour cette raison qu'on en fait la cible privilégiée des interventions psychosociales contemporaines. En outre, comment peut-on prendre position contre un programme qui se donne comme objectif l'amélioration de la santé de la «communauté» ou la satisfaction des «vrais besoins des clients»?

Tout projet d'intervention en santé mentale communautaire ne peut toutefois se réaliser si l'on ne tient pas compte des nombreux «alliés» sur le terrain, considérés désormais à la fois comme des partenaires précieux de l'intervention et comme nouvelle cible de celle-là (entre autres: Poitras et Gendron, 1989). L'intervention qui se veut communautaire devra donc dispenser moins de services directs tout en se concentrant sur le soutien des réseaux «naturels» d'entraide dans l'objectif ultime de remettre au citoyen et à la «communauté» la responsabilité de leur santé. C'est là une rengaine 
que les administrateurs d'un État en crise financière incorporent systématiquement à leur répertoire, davantage soucieux de l'équilibre budgétaire que de l'hétéronomie du citoyen consommateur passif de services publics de santé devenus de plus en plus dispendieux.

S'il est vrai que la critique «communautaire» à l'égard des pratiques « dé-responsabilisantes» de l'État-providence et des approches médicale et psychiatrique d'intervention vise le pouvoir certes démesuré de certaines institutions et des praticiens professionnels sur leurs patients, les intervenants communautaires réclament depuis longtemps, à leur tour, l'élargissement de leur pouvoir d'intervention, mais cette fois au nom de la santé mentale du client et de sa communauté. Car «se situer en fonction d'un pôle négatif seulement, celui de la maladie, limite le pouvoir d'intervention du psychologue et de la psychologue et par conséquent limite la qualité des services» (Côté et al. 1983, p. 11). Étendre les pouvoirs d'intervention des intervenants communautaires et repousser les frontières de l'intervention dans un esprit proactif à des secteurs de la vie sociale qui auraient miraculeusement échappé aux pouvoirs démesurés des intervenants « réactifs» signifie améliorer la qualité des services lorsqu’il s'agit d'un modèle communautaire d'intervention (Guay, 1987).

L'univers du «communautaire» peut être conçu comme une structure de co-animation permanente où tous les membres sont à la fois, ou alternativement, «animés» et «animateurs», selon leurs positions relatives à différents moments de leur vie. La conception du «tissu social» qui sous-tend les interventions d'inspiration communautaire apparaît davantage comme un ensemble de réseaux d'individus à la recherche de l'équilibre, voire de l'harmonie, que comme un espace social conflictuel traversé et organisé par des rapports de force qui transcendent le domaine du "psychosocial» et le réseau social du «client» cible de l'intervention.

Tel qu'on l'a vu dans le cas des interventions d'inspiration cognitivo-comportementale, les références à des problématiques économiques, sociales et politiques plus larges qui pourraient être à l'origine des «difficultés» des personnes "en difficulté» laissent la place centrale encore une fois à l'engagement responsable, constructif, «réaliste» et solidaire des citoyens et citoyennes capables de devenir les véritables maîtres de leur destinée biopsychosociale par le développement optimal de leur capacité d'adaptation et des ressources de «leur communauté».

$* * *$

À l'instar d'autres scènes de la vie des sociétés contemporaines (qu'on pense aux «consensus» des différents sommets socio-économiques, ou encore à la similitude des programmes des partis politiques censés être aux antipodes), une discrète convergence d'objectifs se dégage en filigrane dans l'univers de l'intervention psychosociale et psychothérapeutique, transcendant les querelles d'école et les conflits corporatifs. En effet, l'objectif fondamental d' "aider» les sujets à s'adapter à leurs contextes plutôt qu'à problématiser leurs «expériences de difficulté» semble constituer le point de ralliement 
de ce qu'on pourrait appeler les «agents» de la santé mentale, toutes orientations confondues, ou presque.

La psychiatrie n'est pas la seule (elle ne l'a jamais été d'ailleurs) à osciller entre l'administration de thérapeutiques aux personnes souffrantes et le dressage des sujets, car les modèles d'intervention qui se présentent comme des solutions de rechange progressistes ne contestent pas, du moins pour l'essentiel, la méta-valeur «thérapeutique» et sociale du primat de l'adaptation des sujets à leurs environnements, se concentrant plutôt sur la recherche de moyens « alternatifs » pour y parvenir.

\section{RÉSUMÉ}

Les profondes transformations que le champ des pratiques psychothérapeutiques et psychosociales a subies dans les sociétés occidentales contemporaines au cours des vingt-cinq dernières années ne sauraient être comprises dans le seul cadre d'une analyse des discours théoriques rivaux qui s'y affrontent dans le but de démontrer la pertinence des interventions qu'ils cautionnent. La prise en compte des transformations sociales plus larges qui se sont opérées dans ces sociétés au niveau du mode de régulation des conduites des sujets, nous semble l'une des portes d'entrée privilégiées pour mieux comprendre la nouvelle configuration de ce champ de pratiques. Les rapports existant entre le mode de régulation des conduites des sujets et les interventions psychosociales et psychothérapeutiques sont analysés dans le contexte québécois.

\section{SUM MARY}

The profound transformations which the field of psychotherapeutic and psycho-social practices have undergone in contemporary Western societies over the last twenty-five years can not be understood only within the framework of the analysis of rival theoretical discourses which have confronted each over the relevance of the interventions they stand for. It seems to us that taking into account the broader social transformations that have operated in these societies on the level of the mode of regulation of behaviour is one of the favoured ports of entry for better understanding the new configuration of this field of practice. The relationships existing between the mode of regulation of the behaviour and psycho-social and psychotherapeutic interventions are analysed in the Quebec context.

\section{RESUMEN}

Las profundas transformaciones que el campo de las prácticas sicoterapéuticas y sicosociales sufrió en las sociedades occidentales contemporáneas en el curso de los últimos veinticinco años no pueden comprenderse en el marco de un análisis que se limita a los discursos teóricos rivales que se enfrentan con el objetivo de demostrar la pertinencia de las intervenciones que ellos legitiman. La consideración de las transformaciones sociales que se operaron en estas sociedades en el plano de la évolución del modo de regulación de las conductas de los sujetos nos parece una de la puertas de entrada privilegiadas para comprender la nueva configuración de este campo de prácticas. Las relaciones que existen entre el modo de regulación de conductas de los sujetos y las intervenciones sicosociales y sicoterapeúticas son analizadas en el contexto quebequense. 


\section{BIBLIOGRAPHIE}

Alain, M. et Y. Lussier (1988), «Impact psychologique de la séparation et du divorce», Santé mentale au Québec, vol. 13, nº 1, p. 57-68.

Bandura, A. (1993), «La théorie sociale-cognitive des buts», Revue Québécoise de Psychologie, vol. 14, nº 2, p. 43-84.

Bergin, A. E. et S. Garfield (1994), Handbook of psychotherapy and behavior change, New York, Wiley.

Boivert, J. et M. BeAudry (1984), «Pour une validation des instruments d'auto...», Revue Québécoise de Psychologie, vol. 5, $\mathrm{n}^{\circ}$ 3, p. 44-47.

Bordeleau, Y. (1998), Carrière de consultant, Montréal, ams Éditeur.

Bouchard, J.-M. (1986), «Désinstitutionnalisation, «communautarisation des services et formation des intervenants», Santé mentale au Québec, vol. 11, nº 2, p. 26-36.

Bouffard, L. (1993), «Présentation: buts et perspective future», Revue Québécoise de Psychologie, vol. 14, n 1, p. 61-68.

Bouffard, L. et E. Bastin (1993), «Les hauts et les bas des perspectives d'avenir au cours de la vie», Revue Québécoise de Psychologie, vol. 14, $\mathrm{n}^{\circ}$ 3, p. 53-76.

Brillon, M. (1997), «Or pur ou vil plomb? La fonction soignante de l'analyste», Revue Québécoise de Psychologie, vol. 18, $\mathrm{n}^{\circ}$ 1, p. 103-114.

CAMPAGNA, J.-L. (1981), «La prévention en santé mentale communautaire», Santé mentale au Québec, vol. $6, \mathrm{n}^{\circ} 1, \mathrm{p} .19-29$.

Caplan, G. (1964), Principles of Preventive Psychiatry, New York, Basic Books.

Castel, R. (1995), Les métamorphoses de la question sociale. Une chronique du salariat, Paris, Fayard.

Castel, R. (1981), La gestion des risques: de l'anti-psychiatrie à l'après-psychanalyse, Paris, Les Éditions de Minuit.

Castel, R., F. Castel et A. Lovell (1979), La société psychiatrique avancée, le modèle américain, Paris, Grasset.

CAstoriadis, C. (1991), «Le délabrement de l'Occident», Esprit, décembre, pp. 36-54.

Côté, P. et al. (1983), «Santé mentale et interventions: nouveaux défis pour les psychologues», Revue Québécoise de Psychologie, vol. 4, nº 1, p. 4-12.

Cousineau, P. (1995), «Les approches cognitives. Présentation», Revue Québécoise de Psychologie, vol. 16, n 2, p. 51-54.

DAvid, H. (1997), «La psychanalyse en questions», Revue Québécoise de Psychologie, vol. 18, nº 1, p. 175-180.

De Caravalho, R. J., (1990), "A History of the "Third Force" in Psychology», Journal of Humanistic Psychology, vol. 30, no 4, p. 22-44.

Donzelot, J. (1994), L'État animateur, essai sur la politique de la ville, Paris, Éditions Esprit.

Donzelot , J. (1977), La police des familles, Paris, Éditions de Minuit.

Doucet, P. et W. Reid (dir.) (1996), La psychothérapie psychanalytique, Boucherville, Gaëtan Morin.

Duruz, N. (1994), Psychothérapie ou psychothérapies?, prolégomènes à une analyse comparative, Lausanne, Delachaux et Niestlé.

Ehrenberg, A. (1998), La fatigue d'être soi, dépression et société, Paris, Odile Jacob.

Ehrenberg , A. (1995), L'individu incertain, Paris, Calmann-Lévy.

Fernandez, L. C. (1997), «Misère marchande», Psychologie Québec, novembre, p. 13-14.

Foucault, M. (1984), «Deux essais sur le sujet du pouvoir», in H. DREYFUS et R. RABINOW, Michel Foucault. Un parcours philosophique, Paris, Gallimard, p. 297-321.

Foucault, M. (1976) Histoire de la sexualité I, La volonté de savoir, Paris, Gallimard.

GARFIELD, S. L. (1984), «Le rapprochement et l'éclectisme en psychothérapie», Revue Québécoise de Psychologie, vol. 5, $\mathrm{n}^{\circ} 1, \mathrm{p} .55-67$.

Garneau, J. (1984), «L'autodéveloppement: une stratégie d'instrumentation », Revue Québécoise de Psychologie, vol. 5, $\mathrm{n}^{\circ}$ 3, p. 48-59. 
Gendreau, C. (1998), «Le public, le privé, le communautaire», Santé mentale au Québec, vol. 13, nº 1, p. 48-56.

Gendron, C. et L. Poitras (1989), «La prise en charge de la famille: problématique et implication de cette politique en psychogériatrie», Santé mentale au Québec, vol. 14, nº 1, p. 179-190.

GuATtARI, F. (1993), préface de l'ouvrage de N. SinelnikofF, Les psychothérapies, Inventaire critique, Paris, ESF Éditeur, p. 5-8.

GuAY, J. (1987), «La psychologie communautaire offre-t-elle une voie d'avenir à la psychologie clinique?», Revue Québécoise de Psychologie, vol. 8, nº 2, p. 101-120.

Hade-Hétu, L. (1978), «Rendez-vous avec Thérèse Bertherat», Santé mentale au Québec, vol. 3, nº 1, p. 97-100.

KristeVa, J. (1993), Les nouvelles maladies de l'âme, Paris, Fayard, 1993.

Kuehlwein, K. T. et H. Rosen (1993), Cognitive Therapies in Action: Evolving Innovative Practice, San Francisco, Jossey-Bass.

LAsch, C. (1977), The Culture of Narcissism: American Life in an Age of Diminishing Expectations, New York, Warner.

LAurin, N. (1999), «Le démantèlement des institutions intermédiaires de la régulation sociale. Vers une nouvelle forme de domination ", Sociologie et sociétés, vol. 31, n 2, p. 65-72.

Lebeu A. et al. (1987), "Analyse exploratoire de pratiques de santé mentale en situation de chômage: le cas d'ouvriers et d'ouvriers du secteur manufacturier de l'Est de Montréal», Santé mentale au Québec, vol. $12, \mathrm{n}^{\circ} 2$, novembre, p. 47-63.

Lipovetsky, G. (1983), L’ère du vide, Essais sur l'individualisme contemporain, Paris, Gallimard, et (1993) pour la postface.

MaUrice (1995), «Les actes réservés en psychologie: un outil essentiel à la protection du public», Psychologie Québec, vol. 12, nº 4.

Morissette, L. et M. Riel (1984), Guide des nouvelles thérapies, Québec, Québec Science Éditeur.

NADEAU, B. (1983), «La prévention en santé communautaire... Quelques réflexions», Revue Québécoise de Psychologie, vol. 4, $\mathrm{n}^{\circ}$ 1, février, p. 13-20.

Nathan, T. et I. Stengers (1995), Médecins et sorciers, Le Plessis-Robinson, Synthélabo éditeur.

Otero, М. (1999), «L'intervenant psychosocial et sa "magie” », Revue canadienne de psycho-éducation, vol. 28, $\mathrm{n}^{\circ} 1$, p. 105-114.

Отеro, M. (2000), Du psychanalysme à l'impératif de l'adaptation: un nouveau mode de régulation des conduites des sujets. Une analyse de trois revues québécoises de psychologie de 1977 à 1997, thèse de doctorat, département de sociologie, Université de Montréal.

Paquette, C. (1984), «D’une pédagogie ouverte à l'autodéveloppement», Revue Québécoise de Psychologie, vol. $5, \mathrm{n}^{\circ} 3$, p. 91-103.

Perreault, N. et H. Béguin (1994), «Prévention en santé mentale: quand les mots de l'évaluation ne suffisent pas pour le dire», Revue Québécoise de Psychologie, vol. 15, nº 2 , p. 153-170.

Piotte, M. et A. Duchesne (1977), «Thérapie du sentiment», Santé mentale au Québec, vol. 2, nº 2, p. 92-102. Richard, H. (1997), «Psychanalyse et postmodernité: la psychanalyse en procès?», Revue Québécoise de Psychologie, vol. 18, $\mathrm{n}^{\circ}$ 1, p. 83-102.

Royer, D. (1977), «L'analyse bioénergétique», Santé mentale au Québec, vol. 2, nº 1, p. 13-20

Sennett, R. (1977), The Fall of Public Man, New York, Knopf.

Sinelnikoff, N. (1993), Les psychothérapies, Inventaire critique, Paris, ESF.

St-Arnaud, Y. (1986), «La prise en charge de ses relations interpersonnelles», Revue Québécoise de Psychologie, vol. 7, $\mathrm{n}^{\circ}$ 1-2, p. 11-25.

St-Arnaud , Y. (1984), «Une table ronde sur l'autodéveloppement », Revue Québécoise de Psychologie, vol. 5, $\mathrm{n}^{\circ} 3, \mathrm{p} .30-40$. 